\title{
An Analysis and Design for Nonlinear Quadratic Systems Subject to Nested Saturation
}

\author{
Minsong Zhang' and Fu Chen ${ }^{2}$ \\ ${ }^{1}$ School of Mathematics and Computer Science, Hubei University of Arts and Science, Xiangyang, Hubei 441053, China \\ ${ }^{2}$ School of Automation, Nanjing University of Science and Technology, Nanjing, Jiangsu 210094, China \\ Correspondence should be addressed to Minsong Zhang; minsongzhang0313@163.com
}

Received 24 June 2013; Accepted 26 August 2013

Academic Editor: Vu Phat

Copyright (c) 2013 M. Zhang and F. Chen. This is an open access article distributed under the Creative Commons Attribution License, which permits unrestricted use, distribution, and reproduction in any medium, provided the original work is properly cited.

This paper considers the stability problem for nonlinear quadratic systems with nested saturation input. The interesting treatment method proposed to nested saturation here is put into use a well-established linear differential control tool. And the new conclusions include the existing conclusion on this issue and have less conservatism than before. Simulation example illustrates the effectiveness of the established methodologies.

\section{Introduction}

In the past decades, there has been significant interest in the study of the quadratic systems due to such systems' widely present from engineering systems to economic phenomena [1-5]. To some extent, such systems can accurately describe the interaction dynamics of various species, for example, in enzyme kinetics [6] or population models [7]. In many practical control applications, saturation nonlinearities almost emerge everywhere in the real control process. In particular, the input saturation is an impactive source resulting in instability of the control systems. Numerous researches on the various control problems containing saturation nonlinearity have been conducted; for example, see $[8,9]$ and the references therein. In general, we have two primary approaches to deal with saturation nonlinearity; one is to deal with the saturation nonlinearity as a local sector bound nonlinearity with different multipliers $[10,11]$; the other $[12,13]$ is to conduct the saturation nonlinearity as a polytopic representation, which reduces less conservatism than the first one.

Over the years, several papers have focused on the study of the quadratic systems subject to saturation input. The region of attraction (RA) is an interesting issue in the stability analysis of nonlinear quadratic systems. A Lyapunov-based procedure is presented in [14] to compute an ellipsoidal estimate of the RA of a second-order nonlinear systems containing either linear and quadratic or linear and cubic terms. More recently, the problem of estimating the RA of quadratic systems subject to saturation input has been solved as a transformative linear matrix inequalities (LMIs) feasibility problem $[15,16]$, although in practice it is sometimes fairly difficult to construct an appropriate Lyapunov function. References $[17,18]$ consider linear systems subject to nested actuator saturation and extend the corresponding conditions.

Based on the Lyapunov function and a particular presentation for the quadratic terms, the purpose of this paper is in a sense to be made precise of the sufficient conditions for local stabilization for the quadratic systems subject to nested saturation input in terms of LMIs. Our research is provoked by the work of [19]. It is clear that the sufficient conditions presented in [4] are special cases of our newly built treatment of nest saturation nonlinearity. Moreover, this paper is organized as follows. The system discussed is presented in detail in Section 2. Section 3 states the main results of the local stabilization for the quadratic systems in terms of LMIs. An example is given in Section 4 to illustrate the proposed methodologies, and Section 5 concludes the whole paper. 
Notation. Throughout this paper, standard notation will be adopted. For a matrix $P, P>(\geq) 0$ means that $P$ is a symmetric and (semi-) positive definite matrix, and $\mathscr{E}(P)=\left\{x \in R^{n}\right.$ : $\left.x^{T} P x \leq 1\right\}$. For a matrix $H \in R^{m \times n}$, we define $\mathscr{L}(H)=\{x \in$ $\left.R^{n}:\|H x\|_{\infty} \leq 1\right\}$, where $\|v\|_{\infty}$ denotes the $\infty$-norm of the vector $v$. The symbol $\mathrm{He}(A)$ denotes $A^{T}+A$, where $A^{T}$ is the transpose of $A ; \lambda_{i}(A)$ denotes the $i$ th eigenvalue of $A$, and $\lambda(A)$ denotes the eigenvalue set of matrix $A$. If $P \geq 0, \lambda_{\text {max }}(P)$ and $\lambda_{\text {min }}(P)$ mean the maximal and minimal eigenvalues of $P$, respectively. Moreover, $F^{i}$ denotes the $i$ th row of matrix $F$, and $B^{(i)}$ denotes the $i$ th diagonal element of the diagonal matrix $B . A \otimes B$ means the Kronecker product of the pair of $(A, B)$. In addition, we need some special notation to present the main results. For a given integer $m \geq 1$, we define $\Phi_{m}=$ $\{1,2, \ldots, m\}, \mathscr{V}_{m}=\left\{\mathscr{V}_{s}: \mathscr{V}_{s} \subset \Phi\right\}$. It is easy to see that there are $2^{m}$ elements in the set $\mathscr{V}_{m}$. Denote the number of elements in $\mathcal{S} \in \mathscr{V}_{m}$ by $\sigma(\mathcal{S})$. Assume that $\sigma(\emptyset)=0$; then, for arbitrary $\mathcal{S} \in \mathscr{V}_{m}$, we can denote $\mathcal{S}=\left\{i_{1}, i_{2}, \ldots, i_{\sigma(\mathcal{S})}\right\}$ with $1 \leq i_{1}<i_{2}<\cdots<i_{\sigma(\mathcal{S})} \leq m[18]$.

Definition 1 (see [18]). Let $\mathcal{S}=\left\{i_{1}, i_{2}, \ldots, i_{\sigma(\mathcal{S})}\right\} \in \mathscr{V}_{m}$ with $1 \leq i_{1}<i_{2}<\cdots<i_{\sigma(\mathcal{S})} \leq m$. Define $H_{\mathcal{S}} \in R^{m \times n}$ as a matrix such that the $i_{j}$ th rows of $H_{\mathcal{S}}$ are nonzero while the other rows are zeros, and define $D_{\mathcal{S}}$ as a diagonal matrix such that the $i_{j}$ th diagonal elements are 1 and the others are zeros. Furthermore, define $D_{\mathcal{\delta}}^{-}=I_{m}-D_{\mathcal{\delta}}$. For two integers $m \geq 1$ and $q \geq 1$, one has

$$
\mathscr{W}=\left\{\omega=\left[\begin{array}{llll}
\omega_{1} & \omega_{2} & \cdots & \omega_{m}
\end{array}\right]^{T}\right\}: \omega_{i} \in\{0,1, \ldots, q\} .
$$

Definition 2 (see [18]). Let $\omega \in \mathscr{W}$ be a given vector, and denote the $i$ th element of $\omega$ by $\omega_{i}$. The matrix $\mathscr{H}_{\omega} \in R^{m \times n}$ is defined as $\mathscr{H}_{\omega}^{i}=0$ if $\omega_{i}=0$, for all $i \in \Phi_{m}$.

\section{Plant Analysis}

Consider the quadratic system subject to nested saturatedinput:

$$
\dot{x}(t)=A x(t)+\left(\begin{array}{c}
x(t)^{T} A_{q 1} x(t) \\
x(t)^{T} A_{q 2} x(t) \\
\vdots \\
x(t)^{T} A_{q n} x(t)
\end{array}\right)+B \text { sat }(u(t)),
$$

where $x(t) \in R^{n}, u=F x$ is the control input, $F \in R^{m \times n}$ is a given matrix, and $\operatorname{sat}(u)$ denotes the nested saturation nonlinearity of $u$. Also $A \in R^{n \times n}, B \in R^{n \times m}$, and $A_{q i} \in R^{n \times n}$, $i=1, \ldots, n$. Define matrices $\mathscr{A}_{q 0} \in R^{n \times n^{2}}$ as follows:

$$
\mathscr{A}_{q 0}=\left(\begin{array}{cccc}
A_{q 1(1)} & A_{q 1(2)} & \cdots & A_{q 1(n)} \\
\vdots & \vdots & \ddots & \vdots \\
A_{q n(1)} & A_{q n(2)} & \cdots & A_{q n(n)}
\end{array}\right)
$$

where $A_{q i(j)} \in R^{1 \times n}$ denotes the $j$ th row of matrix $A_{q i} \in R^{n \times n}$. The quadratic system (2) can be read as

$$
\dot{x}(t)=\left(A+\mathscr{A}_{q 0}\left(I_{n} \otimes x(t)\right)\right) x(t)+B \operatorname{sat}(u(t)) .
$$

In the next section, we consider three types of $\operatorname{sat}(u(t))$ and draw the corresponding conclusions for the quadratic systems (4). The following lemmas are essential for the development of our paper.

Lemma 3 (see [15]). Consider matrix $P \in R^{n \times n}, P=P^{T}>0$, and a vector $v$ such that $\|v\|=1$. Every point on the boundary of an ellipsoid, $\partial \mathscr{E}(P)=\left\{x \in R^{n}: x^{T} P x=1\right\}$, can be parameterized by $x=P^{-1 / 2} U v$ where $U^{T} U=I$.

Lemma 4 (see [19]). Let $a, b \in R$ be two arbitrary scalars; then

$$
a \text { sat }(b) \leq \max \{a b,-|a|\} \text {. }
$$

Lemma 5 (see [18]). Let $F \in R^{n \times m}$ be a given matrix and $u \in$ $R^{m}$ an arbitrary vector. If $x \in \mathscr{L}(H)_{\mathcal{S}}$, for all $\mathcal{S} \in \mathscr{V}_{m}$, where $\mathcal{S}, \mathscr{V}_{m}$, and $H_{\mathcal{S}} \in R^{m \times n}$ are defined in Definition 1, then

$$
2 x^{T} F \text { sat }(u) \leq \max _{\mathcal{S} \in \mathscr{V}_{m}}\left\{2 x^{T} F D_{\mathcal{\delta}}^{-} u+x^{T}\left(\operatorname{He}\left(F H_{\mathcal{S}}\right)\right) x\right\} \text {. }
$$

Lemma 6 (see [18]). For two vectors $u, v \in R^{m}$, if $\|v\|_{\infty} \leq$ 1 , then sat $(u) \in \operatorname{co}\left\{D_{\mathcal{\delta}}^{-} u_{i}+D_{\mathcal{\delta}} v_{i}, \mathcal{S} \in \mathscr{V}_{m}\right\}$, where co $\{\cdot\}$ denotes the convex hull of a set.

\section{Main Results}

In this section, through the new treatment of saturation nonlinearity given in Lemma 5, we divide three cases to estimate the RA for the quadratic system (4) subject to nested input saturation with conditional control design.

At first, we consider the quadratic systems subject to conventional actuator saturation. For a saturation level $\mu\left(>\varepsilon_{1}\right)$, the standard saturation function is defined as follows:

$$
[\operatorname{sat} u(t)]_{l} \triangleq \begin{cases}{[u(t)]_{l}} & \text { if }\left|[u(t)]_{l}\right|<\mu \\ \mu & \text { if }[u(t)]_{l} \geq \mu \\ -\mu & \text { if }[u(t)]_{l} \leq-\mu\end{cases}
$$

and it meets the following sufficient condition.

Proposition 7. If there exist a positive scalar $\varepsilon$ and matrices $P>0 \in R^{n \times n}$ and $H_{\mathcal{S}} \in R^{m \times n}$, for all $\mathcal{S} \in \mathscr{V}_{m}$, satisfying the following inequality:

$$
\operatorname{He}\left(\left[\begin{array}{cc}
P\left(A+B D_{\mathcal{S}}^{-} F+B H_{\mathcal{S}}\right)+\frac{\varepsilon}{2} I & P \mathscr{A}_{q 0} \\
0 & -\frac{\varepsilon}{2}\left(I_{n} \otimes P\right)
\end{array}\right]\right)<0 \text {, }
$$

then the region $\mathscr{E}(P) \subseteq \mathscr{L}\left(H_{\mathcal{S}}\right)$ is an estimate of the $R A$ for the nonlinear quadratic system (4) with conditional actuator saturation (7).

Proof. Consider the quadratic Lyapunov function $V(x)=$ $x^{T} P x$, where $P=P^{T}>0$. Let $x \in \mathscr{L}\left(H_{\mathcal{S}}\right)$, for all $\mathcal{S} \in \mathscr{V}_{m}$. 
Then by Lemma 5, the time derivative of $V(x)$ along the trajectories of system (4) is given as

$$
\begin{aligned}
\dot{V}(x)= & x^{T}\left(A^{T} P+P A+P \mathscr{A}_{q 0}\left(I_{n} \otimes x(t)\right)\right. \\
& \left.+\left(I_{n} \otimes x(t)\right)^{T} \mathscr{A}_{q 0}^{T} P\right) x+2 x^{T} P B \operatorname{sat}(F x) \\
\leq & x^{T}\left(A^{T} P+P A+P \mathscr{A}_{q 0}\left(I_{n} \otimes x(t)\right)\right. \\
& \left.+\left(I_{n} \otimes x(t)\right)^{T} \mathscr{A}_{q 0}^{T} P\right) x \\
& +\max _{\delta \in \mathscr{V}_{m}}\left\{2 x^{T} P B D_{\mathcal{\delta}}^{-} F x+x^{T}\left(\operatorname{He}\left(P B H_{\mathcal{S}}\right)\right) x\right\} \\
= & x^{T} \operatorname{He}_{2}\left(P A+P \mathscr{A}_{q 0}\left(I_{n} \otimes P\right)^{-1 / 2}\left(I_{n} \otimes U\right)\left(I_{n} \otimes v\right)\right) x \\
& +\max _{\delta \in \mathscr{V}_{m}}\left\{2 x^{T} P B D_{\mathcal{S}}^{-} F x+x^{T}\left(\operatorname{He}\left(P B H_{\mathcal{S}}\right)\right) x\right\}
\end{aligned}
$$

with $\|v\|^{2}=1$. To guarantee that $\dot{V}(x)<0$ at each point of $\partial \mathscr{E}(P)$, it suffices to verify the following inequality:

$$
\begin{aligned}
\operatorname{He}\{ & P\left(A+B D_{\mathcal{\delta}}^{-} F+B H_{\mathcal{S}}\right) \\
& \left.+P \mathscr{A}_{q 0}\left(I_{n} \otimes P\right)^{-1 / 2}\left(I_{n} \otimes U\right)\left(I_{n} \otimes v\right)\right\}<0 .
\end{aligned}
$$

One can write

$$
\begin{aligned}
\mathrm{He}\{ & \left.P \mathscr{A}_{q 0}\left(I_{n} \otimes P\right)^{-1 / 2}\left(I_{n} \otimes U\right)\left(I_{n} \otimes v\right)\right\} \\
< & \frac{1}{\varepsilon} P \mathscr{A}_{q 0}\left(I_{n} \otimes P\right)^{-1} \mathscr{A}_{q 0}^{T} P \\
& +\varepsilon\left(I_{n} \otimes v\right)^{T}\left(I_{n} \otimes U\right)^{T}\left(I_{n} \otimes U\right)\left(I_{n} \otimes v\right),
\end{aligned}
$$

where $\varepsilon>0$ and

$$
\left(I_{n} \otimes U\right)^{T}\left(I_{n} \otimes U\right)=I_{n^{2}}, \quad\left(I_{n} \otimes v\right)^{T}\left(I_{n} \otimes v\right)=I_{n} .
$$

Then one get

$$
\begin{aligned}
\mathrm{He}( & \left.P \mathscr{A}_{q 0}\left(I_{n} \otimes P\right)^{-1 / 2}\left(I_{n} \otimes U\right)\left(I_{n} \otimes v\right)\right) \\
< & \frac{1}{\varepsilon} P \mathscr{A}_{q 0}\left(I_{n} \otimes P\right)^{-1} \mathscr{A}_{q 0}^{T} P+\varepsilon I .
\end{aligned}
$$

Thus if the inequality

$$
\begin{aligned}
\operatorname{He}( & \left.P\left(A+B D_{\mathcal{S}}^{-} F+B H_{\mathcal{S}}\right)\right) \\
& +\frac{1}{\varepsilon} P \mathscr{A}_{q 0}\left(I_{n} \otimes P\right)^{-1} \mathscr{A}_{q 0}^{T} P+\varepsilon I<0
\end{aligned}
$$

holds, then inequality (10) is satisfied.

If the relation (8) is right, obtained from (14) by the Schur complement, it follows that (10) holds for every $U$ and $v$ satisfying (12). From Lemma 3, if (10) is satisfied, then, for every $x \in \partial \mathscr{E}(P)$, we have $\dot{V}(x)<0$. As $\mathscr{E}(P)$ is a convex set and from the fact that terms $x=P^{-1 / 2} U v$ appear linearly in matrix $\mathrm{He}\left(P\left(A+B D_{\mathcal{S}}^{-} F+B H_{\mathcal{S}}\right)+P \mathscr{A}_{q 0}\left(I_{n} \otimes x(t)\right)\right)$, any point in $\mathscr{E}(P)=\left\{x \in R^{n}: x^{T} P x \leq 1\right\} \subseteq \mathscr{L}\left(H_{\mathcal{S}}\right)$, for all $\mathcal{S} \in \mathscr{V}_{m}$, also satisfies $\dot{V}(x)<0$, and thus $\mathscr{E}(P)$ is an estimate of the RA for the quadratic system (4) with conventional actuator saturation (7). The proof is completed.

In the following, we consider the quadratic systems subject to diagonal nested actuator saturation:

$$
\dot{x}(t)=\left(A+\mathscr{A}_{q}\left(I_{n} \otimes x(t)\right)\right) x(t)+B \text { sat }(u(t)),
$$

where $B=\left[b_{1}, b_{2}, \ldots, b_{m}\right] \in R^{n \times m} \cdot u(t)$ is the nested saturation input function as follows:

$$
\begin{aligned}
u(t)= & F_{q-1} x(t)+B_{q-1} \text { sat } \\
& \times\left(F_{q-2} x(t)+\cdots\right. \\
& \left.\quad+B_{2} \operatorname{sat}\left(F_{1} x(t)+B_{1} \operatorname{sat}\left(F_{0} x(t)\right)\right) \cdots\right)
\end{aligned}
$$

in which $B_{i} \in R^{m \times m}, i \in\{1,2, \ldots, q-1\}$, are diagonal matrices and $F_{j} \in R^{m \times n}, j \in\{0,1, \ldots, q-1\}$, are feedback gain matrices.

To express concisely, we denote

$$
\begin{aligned}
& \left\{\begin{array}{l}
\mathscr{B}_{q}=I, \\
\mathscr{B}_{q-1}=B_{q-1}, \\
\mathscr{B}_{q-2}=B_{q-1} B_{q-2}, \\
\vdots \\
\mathscr{B}_{1}=B_{q-1} B_{q-2}, \ldots, B_{2} B_{1}, \\
\mathscr{B}_{0}=0,
\end{array}\right. \\
& \left\{\begin{array}{l}
\mathscr{F}_{q}=0, \\
\mathscr{F}_{q-1}=F_{q-1}, \\
\mathscr{F}_{q-2}=F_{q-1}+\sum_{i=q-2}^{q-2} \mathscr{B}_{i+1} F_{i}, \\
\vdots \\
\& \mathscr{F}_{0}=F_{q-1}+\sum_{i=0}^{q-2} \mathscr{B}_{i+1} F_{i} .
\end{array}\right.
\end{aligned}
$$

Then we propose a new result for the local stability of the quadratic system (15).

Theorem 8. If there exist a positive scalar $\varepsilon$ and matrices $P>0 \in R^{n \times n}$, and $\mathscr{H}_{\omega} \in R^{m \times n}$, for all $\omega \in \mathscr{V}$, defined in Definition 2, such that

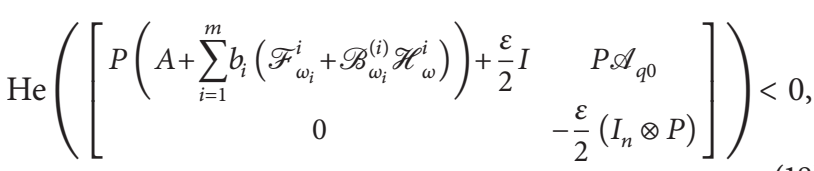

then the quadratic system (15) is locally asymptotically stable and $\mathscr{E}(P) \subseteq \mathscr{L}\left(\mathscr{H}_{\omega}\right)$ is an estimate of the $R A$. 
Proof. Consider the Lyapunov quadratic function $V(x)=$ $x^{T} P x$ with $P=P^{T}>0$. Its time derivative along the trajectories of the system (15) is given by

$$
\begin{aligned}
\dot{V}(x)= & x^{T} \operatorname{He}\left(P A+P \mathscr{A}_{q 0}\left(I_{n} \otimes x(t)\right)\right) x \\
& +\sum_{i=1}^{m} 2 x^{T} P b_{i} \operatorname{sat}\left(\phi_{j-1}^{i}\right),
\end{aligned}
$$

where $j \in \Phi_{q} \backslash\{1\}$. We define $\phi_{j-1}^{i}=F_{j-1}^{i} x+B_{j-1}^{(i)} \rho_{j-1}^{i}$ with $\rho_{j-1}^{i}=\operatorname{sat}\left(F_{j-2}^{i} x+\cdots+B_{2} \operatorname{sat}\left(F_{1} x(t)+B_{1} \operatorname{sat}\left(F_{0} x(t)\right)\right) \cdots\right)$. From Lemma 4, we have

$$
\begin{aligned}
2 x^{T} P b_{i} \text { sat }\left(\phi_{j-1}^{i}\right) & \leq \max \left\{2 x^{T} P b_{i} \phi_{q-1}^{i},-2\left|x^{T} P b_{i}\right|\right\}, \\
2 x^{T} P b_{i} B_{q-1}^{(i)} \rho_{q-1}^{i} & =2 x^{T} P b_{i} B_{q-1}^{(i)} \operatorname{sat}\left(\phi_{q-2}^{i}\right) \\
& \leq \max \left\{2 x^{T} P b_{i} B_{q-1}^{(i)} \phi_{q-2}^{i},-2\left|x^{T} P b_{i} B_{q-1}^{(i)}\right|\right\} .
\end{aligned}
$$

Repeating the above procedure and substituting (21) into (20) yield

$$
\begin{gathered}
2 x^{T} P b_{i} \operatorname{sat}\left(\phi_{q-1}^{i}\right) \\
\leq \max \left\{2 x^{T} P b_{i} \mathscr{F}_{0}^{i} x, 2 x^{T} P b_{i} \mathscr{F}_{1}^{i} x-2\left|x^{T} P b_{i} \mathscr{B}_{1}^{(i)}\right|, \ldots,\right. \\
\left.2 x^{T} P b_{i} \mathscr{F}_{q-1}^{i} x-2\left|x^{T} P b_{i} \mathscr{B}_{q-1}^{(i)}\right|,-2\left|x^{T} P b_{i}\right|\right\} \\
=\max \left\{2 x^{T} P b_{i} \mathscr{F}_{0}^{i} x-2\left|x^{T} P b_{i} \mathscr{B}_{0}^{(i)}\right|,\right. \\
2 x^{T} P b_{i} \mathscr{F}_{1}^{i} x-2\left|x^{T} P b_{i} \mathscr{B}_{1}^{(i)}\right|, \ldots, \\
2 x^{T} P b_{i} \mathscr{F}_{q-1}^{i} x-2\left|x^{T} P b_{i} \mathscr{B}_{q-1}^{(i)}\right|, \\
\left.2 x^{T} P b_{i} \mathscr{F}_{q}^{i} x-2\left|x^{T} P b_{i} \mathscr{B}_{q}^{(i)}\right|\right\} .
\end{gathered}
$$

Let $\omega \in \mathscr{W}$ be an arbitrary vector. Then it follows from (22) that

$$
\begin{aligned}
& \sum_{i=1}^{m} 2 x^{T} P b_{i} \text { sat }\left(\phi_{q-1}^{i}\right) \\
& \quad \leq \max _{\delta \in \mathscr{V}_{m}}\left\{\sum_{i=1}^{m} 2 x^{T} P b_{i} \mathscr{F}_{\omega_{i}}^{i} x-2\left|x^{T} P b_{i} \mathscr{B}_{\omega_{i}}^{(i)}\right|\right\} .
\end{aligned}
$$

Assume that $x \in \mathscr{L}\left(\mathscr{H}_{\omega}\right)$, for all $\omega \in \mathscr{W}$. For $\mathscr{E}(P) \subseteq$ $\mathscr{L}\left(\mathscr{H}_{\omega}\right)$, we obtain the following inequality from (23) and the property of $\mathscr{L}\left(\mathscr{H}_{\omega}\right)$ :

$$
\begin{aligned}
& \sum_{i=1}^{m} 2 x^{T} P b_{i} \text { sat }\left(\phi_{q-1}^{i}\right) \\
& \quad \leq \max _{\mathcal{S} \in \mathscr{V}_{m}}\left\{\sum_{i=1}^{m} x^{T}\left(\operatorname{He}\left(P b_{i}\left(\mathscr{F}_{\omega_{i}}^{i}+\mathscr{B}_{\omega_{i}}^{(i)} \mathscr{H}_{\omega}^{i}\right)\right)\right) x\right\} .
\end{aligned}
$$

Therefore, if (18) is satisfied, we deduce from (20) and (24) that $\dot{V}(x) \leq-\varepsilon\|x\|^{2}$, for all $x \in \mathscr{L}\left(\mathscr{H}_{\omega}\right)$, for all $\omega \in \mathscr{W}$, where $\omega>0$ is a sufficiently small scalar; namely, $\mathscr{E}(P) \subseteq \mathscr{L}\left(\mathscr{H}_{\omega}\right)$, for all $\omega \in \mathscr{W}$, is a contractively invariant set. The proof is completed.

Finally, we study the estimate of the RA of the quadratic system with general nested saturation as follows:

$$
\dot{x}(t)=\left(A+\mathscr{A}_{q 0}\left(I_{n} \otimes x(t)\right)\right) x(t)+B \text { sat }(u(t)),
$$

where $B_{q} \in R^{n \times m_{q}}$ and

$$
\begin{aligned}
& u(t)=F_{q-1} x(t) \\
& \quad+B_{q-1} \text { sat } \times\left(F_{q-2} x(t)+\cdots\right. \\
& \left.\quad+B_{2} \operatorname{sat}\left(F_{1} x(t)+B_{1} \operatorname{sat}\left(F_{0} x(t)\right)\right) \cdots\right)
\end{aligned}
$$

in which $F_{0} \in R^{m_{1} \times n}, F_{i} \in R^{m_{i+1} \times n}$, and $B_{i} \in R^{m_{i+1} \times m_{i}}, i \in \Phi_{q-1}$, the matrices $B_{i}, i \in \Phi_{q-i}$, are not necessarily diagonal, and $m_{i}$, $i \in \Phi_{q}$, are not necessarily the same.

Theorem 9. If there exist a positive scalar $\varepsilon$ and matrices $P=$ $P^{T}>0 \in R^{n \times n}$ and $H_{\mathcal{S}_{i}} \in R^{m_{i} \times n}, i \in \Phi_{q}$, for all $\left(\mathcal{S}_{1}, \mathcal{S}_{2}\right.$, $\left.\ldots, \mathcal{S}_{q}\right) \in\left(\mathscr{V}_{m_{1}} \times \mathscr{V}_{m_{2}} \times \cdots \times \mathscr{V}_{m_{q}}\right)$ such that the following inequality holds:

$$
\operatorname{He}\left(\begin{array}{cc}
P(A+\Xi)+\frac{\varepsilon}{2} I & P \mathscr{A}_{q 0} \\
0 & -\frac{\varepsilon}{2}\left(I_{n} \otimes P\right)
\end{array}\right)<0 \text {, }
$$

where

$$
\begin{aligned}
\Xi= & \sum_{j=1}^{q}\left(\prod_{i=q}^{j} B_{i} D_{\mathcal{S}_{i}}^{-}\right) F_{j-1} \\
& +\sum_{j=1}^{q-1}\left(\prod_{i=q}^{j+1} B_{i} D_{\mathcal{S}_{i}}^{-}\right) B_{j} H_{\mathcal{S}_{j}}+B_{q} H_{\mathcal{S}_{q}},
\end{aligned}
$$

then the quadratic system (25) is locally asymptotically stable for every initial condition belonging to the region $\mathscr{E}(P) \subseteq$ $\mathscr{L}\left(H_{\mathcal{S}_{i}}\right)$.

Proof. Consider the Lyapunov quadratic function $V(x)=$ $x^{T} P x$ with $P=P^{T}>0$. Its time derivative along the quadratic system (25) is given as

$$
\begin{aligned}
\dot{V}(x)=x^{T}( & A^{T} P+P A+P \mathscr{A}_{q 0}\left(I_{n} \otimes x(t)\right) \\
& \left.+\left(I_{n} \otimes x(t)\right)^{T} \mathscr{A}_{q}^{T} P\right) x+2 x^{T} P B_{q} \operatorname{sat}\left(\phi_{j}\right),
\end{aligned}
$$


where, for $j \in \Phi_{q-1}$, we define $\phi_{j}=F_{j} x+B_{j} \rho_{j}$ with $\rho_{j}=$ $\operatorname{sat}\left(F_{j-1} x+\cdots+B_{2} \operatorname{sat}\left(F_{1} x+B_{1} \operatorname{sat}\left(F_{0} x\right)\right) \ldots\right)$. Let $x \in \mathscr{L}\left(\mathscr{H}_{\delta_{i}}\right)$, $\mathcal{S}_{i} \in \mathscr{V}_{m_{i}}$. Then it follows from Lemma 4 that

$$
\begin{gathered}
\dot{V}(x) \leq x^{T} \operatorname{He}\left(P A+P \mathscr{A}_{q 0}\left(I_{n} \otimes x(t)\right)\right) x \\
+\max _{\delta_{q} \in \mathscr{V}_{m_{q}}}\left\{2 x^{T} P B_{q} D_{\mathcal{S}_{q}}^{-} \phi_{q-1}+x^{T} \operatorname{He}\left(P B_{q} H_{\mathcal{S}_{q}}\right) x\right\} \\
=\max _{\mathcal{S}_{q} \in \mathscr{V}_{m_{q}}}\left\{x ^ { T } \operatorname { H e } \left(P B_{q}\left(H_{\mathcal{S}_{q}}+D_{\mathcal{S}_{q}}^{-} F_{q-1}\right)+P A\right.\right. \\
\left.+P \mathscr{A}_{q 0}\left(I_{n} \otimes x(t)\right)\right) x \\
\left.+2 x^{T} P B_{q} D_{\mathcal{S}_{q}}^{-} B_{q-1} \rho_{q-1}\right\}
\end{gathered}
$$

where

$$
\begin{aligned}
& 2 x^{T} P B_{q} D_{\mathcal{S}_{q}}^{-} B_{q-1} \rho_{q-1} \\
& =2 x^{T} P B_{q-1} D_{\delta_{q}}^{-} B_{q-1} \text { sat }\left(\phi_{q-2}\right) \\
& \leq \max _{\mathcal{S}_{q-1} \in \mathscr{V}_{m_{q-1}}}\left\{2 x^{T} P B_{q} D_{\mathcal{S}_{q}}^{-} B_{q-1} D_{\mathcal{S}_{q-1}}^{-} \phi_{q-2}\right. \\
& \left.+x^{T}\left(\operatorname{He}\left(P B_{q} D_{\delta_{q}}^{-} B_{q-1} H_{\delta_{q-1}}\right)\right) x\right\} \\
& =\max _{\mathcal{S}_{q-1} \in \mathscr{V}_{q-1}}\left\{2 x^{T} P B_{q} D_{\mathcal{S}_{q}}^{-} B_{q-1} D_{\mathcal{S}_{q-1}}^{-} B_{q-2} \rho_{q-2}\right. \\
& +x^{T}\left(\operatorname { H e } \left(P B_{q} D_{\delta_{q}}^{-} B_{q-1}\right.\right. \\
& \left.\left.\left.\times\left(H_{\delta_{q-1}}+D_{\delta_{q-1}}^{-} F_{q-2}\right)\right)\right) x\right\} .
\end{aligned}
$$

Then, from the above inequalities, we have that

$$
\begin{aligned}
& \dot{V}(x) \\
& \leq \max _{\mathcal{S}_{q} \in \mathscr{V}_{m_{q}}}\left\{x ^ { T } \operatorname { H e } \left(P B_{q}\left(H_{\mathcal{S}_{q}}+D_{\mathcal{S}_{q}}^{-} F_{q-1}\right)\right.\right. \\
& \left.+P A+P \mathscr{A}_{q 0}\left(I_{n} \otimes x(t)\right)\right) x \\
& +\max _{\mathcal{S}_{q-1} \in \mathscr{V}_{m_{q-1}}}\left\{2 x^{T} P B_{q} D_{\mathcal{S}_{q}}^{-} B_{q-1} D_{\mathcal{S}_{q-1}}^{-} B_{q-2} \rho_{q-2}\right. \\
& +x^{T} \operatorname{He}\left(P B_{q} D_{\mathcal{S}_{q}}^{-} B_{q-1}\right. \\
& \left.\left.\left.\times\left(H_{\mathcal{S}_{q}}+D_{\mathcal{S}_{q-1}}^{-} F_{q-2}\right)\right) x\right\}\right\} \\
& =\max _{\mathcal{S}_{q} \in \mathscr{V}_{m_{q}}} \max _{\mathcal{S}_{q-1} \in \mathscr{V}}\left\{x_{m_{q-1}}^{T} \Xi_{q-1} x+2 x^{T} P B_{q} D_{\mathcal{S}_{q}}^{-} B_{q-1}\right. \\
& \left.\times D_{\delta_{q-1}}^{-} B_{q-2} \rho_{q-2} x\right\},
\end{aligned}
$$

where

$$
\begin{aligned}
\Xi_{q-1}=\operatorname{He}\{ & P B_{q} D_{\mathcal{S}_{q}}^{-} B_{q-1}\left(H_{\mathcal{S}_{q-1}}+D_{\mathcal{S}_{q-1}}^{-} F_{q-2}\right) \\
& +P B_{q}\left(H_{\mathcal{S}_{q}}+D_{\mathcal{S}_{q}}^{-} F_{q-1}\right) \\
& \left.+P A+P \mathscr{A}_{q 0}\left(I_{n} \otimes x(t)\right)\right\} .
\end{aligned}
$$

Repeating the above process, we finally obtain

$$
\begin{aligned}
\dot{V}(x) \leq & \max _{\mathcal{S}_{q} \in \mathscr{V}_{m_{q}} \mathcal{S}_{q-1} \in \mathscr{V}_{m_{q-1}} \cdots \max _{\mathcal{S}_{1} \in \mathscr{V}_{m_{1}}}} \\
& \times\left\{x^{T} \operatorname{He}\left(P\left(A+\mathscr{A}_{q 0}\left(I_{n} \otimes x(t)\right)\right)+\Xi\right) x\right\},
\end{aligned}
$$

where

$$
\begin{aligned}
\Xi= & \operatorname{He}\left(P B\left(H_{\mathcal{S}_{q}}+D_{\mathcal{S}_{q}}^{-} F_{q-1}\right)\right) \\
& +\operatorname{He}\left(P B_{q} D_{\mathcal{S}_{q}}^{-} B_{q-1}\left(H_{\mathcal{S}_{q-1}}+D_{\mathcal{S}_{q-1}}^{-} F_{q-2}\right)\right)+\cdots \\
& +\operatorname{He}\left(P B_{q} D_{\mathcal{S}_{q}}^{-} B_{q-1} D_{\mathcal{\delta}_{q-1}}^{-} B_{q-2} \cdots\right) D_{\mathcal{S}_{2}}^{-} B_{1}\left(H_{\mathcal{S}_{1}}+D_{\mathcal{S}_{1}}^{-} F_{0}\right) .
\end{aligned}
$$

Applying Lemma 3 and (27) for $x \in \partial \mathscr{E}$, each point on the boundary of $\mathscr{E}$, we obtain

$$
\begin{aligned}
\operatorname{He}( & \left.P \mathscr{A}_{q 0}\left(I_{n} \otimes x(t)\right)\right) \\
& =\operatorname{He}\left(P \mathscr{A}_{q 0}\left(I_{n} \otimes P\right)^{-1 / 2}\left(I_{n} \otimes U\right)\left(I_{n} \otimes v\right)\right) \\
& \leq \frac{1}{\varepsilon} P \mathscr{A}_{q 0}\left(I_{n} \otimes P\right)^{-1} \mathscr{A}_{q 0}^{T} P+\varepsilon I,
\end{aligned}
$$

where $\varepsilon>0$ and $U$ and $v$ satisfying (12). Thus if the following inequality holds

$$
\begin{aligned}
\operatorname{He}\left(P\left(A+\Xi+\mathscr{A}_{q} \mathscr{X}\right)\right) \leq \operatorname{He}( & P(A+\Xi) \\
& \left.+\frac{1}{\varepsilon} P \mathscr{A}_{q}\left(I_{n} \otimes P\right)^{-1} \mathscr{A}_{q}^{T} P\right)+\varepsilon I
\end{aligned}
$$$$
<0
$$

then, from the Schur complement, inequality (27) is satisfyied for every $U$ and $v$ satisfying (12). The ellipsoid $\mathscr{E}(P) \subseteq$ $\mathscr{L}\left(H_{\mathcal{S}_{i}}\right)$ is therefore the estimate of the RA for the quadratic system (25).

Remark 10. In the case of $q=1$, Theorem 9 is a set invariance condition on stability analysis for nonlinear quadratic systems subject to traditional saturation input. One can verify that condition (27) is equivalent to the set invariance condition of Proposition 1 presented in [4]; thus we can say that set invariance condition in Theorem 9 here contains the one given in [4]. 


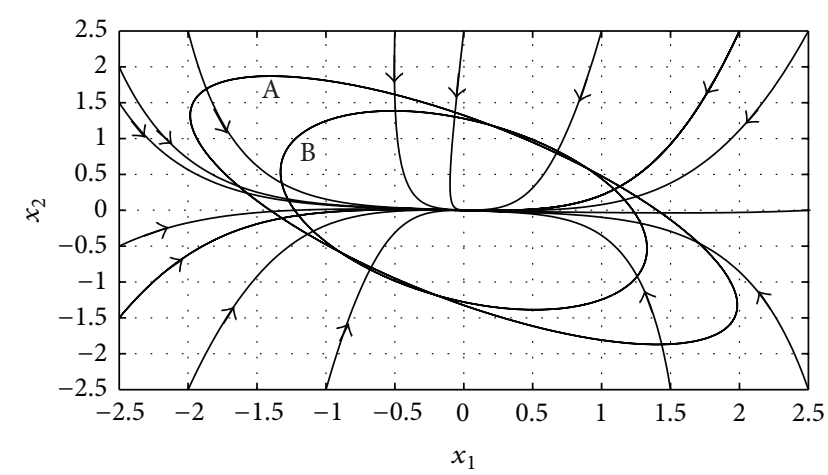

Figure 1: Phase portrait of the system (38) with gains (40) and the estimate of the RA.

\section{Illustrative Example}

In this section, we will present an example to show the effectiveness of the above approaches in Theorem 9. Consider a nonlinear quadratic system in [4] with $q=1$ :

$$
\begin{aligned}
\dot{x}(t)= & \left(A+\mathscr{A}_{q}\left(I_{n} \otimes x(t)\right)\right) x(t) \\
& +B \operatorname{sat}\left(F_{1} x(t)+B_{1} \operatorname{sat}\left(F_{0} x(t)\right)\right),
\end{aligned}
$$

where the coefficient matrices are given as follows:

$$
\begin{aligned}
& A=\left(\begin{array}{ll}
0 & 1 \\
0 & 0
\end{array}\right), \quad B=\left(\begin{array}{cc}
-2 & 1 \\
1 & 1
\end{array}\right), \\
& B_{1}=\left(\begin{array}{cc}
0.2 & 0 \\
0 & 0.1
\end{array}\right), \quad \mathscr{A}_{q}=\left(\begin{array}{lllc}
0 & 0 & 0 & 0.2 \\
2 & 1 & 1 & 0
\end{array}\right) .
\end{aligned}
$$

To measure the impact of the quadratic term of control law on the RA, we compare the value of $\operatorname{trace}(P)$ obtained using the conditions from Theorem 9 , resulting in a control law having gains $K$ and $K_{0}$. The RA of such an equilibrium point obtained by applying Theorem 9 is given by

$$
\begin{gathered}
P=\left[\begin{array}{cc}
0.5889 & -0.1651 \\
-0.1651 & 0.1259
\end{array}\right], \quad K=\left[\begin{array}{ll}
-0.1083 & -1.3299
\end{array}\right] \\
K_{0}=\left[\begin{array}{ll}
0.0760 & -0.0142
\end{array}\right]
\end{gathered}
$$

corresponding to $\lambda=0.0020, \varepsilon=0.1$, and $\eta=0.01$. Figure 1 shows the estimate of the RA of this example with an initial condition $x_{0}=[0.2,0.1]^{T}$.

\section{Conclusion}

In this paper, we have studied the stability of the quadratic systems subject to nested saturation through the polytopic representation of saturation nonlinearity. The interesting treatment of saturation nonlinearity here, which includes the linear differential conclusion, presents more universal results in the form of LMIs through the quadratic Lyapunov functions. Our results effectively reduce the conservatism of the previous study and contain the existing results. The future study will extend the treatment of nested saturation nonlinearity to other control problems and more general classes of the control systems.

\section{Acknowledgment}

This work is supported by the Graduate Innovation and Creativity Foundation of Jiangsu Province under Grant no. CXZZ12-0202.

\section{References}

[1] F. Amato, F. Calabrese, C. Cosentino, and A. Merola, "Stability analysis of nonlinear quadratic systems via polyhedral Lyapunov functions," Automatica, vol. 47, no. 3, pp. 614-617, 2011.

[2] F. Amato, C. Cosentino, and A. Merola, "On the region of attraction of nonlinear quadratic systems," Automatica, vol. 43, no. 12, pp. 2119-2123, 2007.

[3] R. Brockett, Asymptotic Stability and Feedback Stabilization, Birkhauser, Boston, Mass, USA, 1983.

[4] G. Valmórbida, S. Tarbouriech, and G. Garcia, "State feedback design for input-saturating quadratic systems," Automatica, vol. 46, no. 7, pp. 1196-1202, 2010.

[5] G. Scorletti and L. El Ghaoui, "Improved LMI conditions for gain scheduling and related control problems," International Journal of Robust and Nonlinear Control, vol. 8, no. 10, pp. 845877, 1998.

[6] J. D. Murray, Mathematical Biology, vol. 17 of Interdisciplinary Applied Mathematics, Springer, New York, NY, USA, 3rd edition, 2002.

[7] A. J. Lotka, Elements of Physical Biology, Williams and Wilkins, Baltimore, Md, USA, 1925.

[8] D. Coutinho and C. E. de Souza, "Nonlinear state feedback design with a guaranteed stability domain for locally stabilizable unstable quadratic systems," IEEE Transactions on Circuits and Systems, vol. 59, no. 2, pp. 360-370, 2012.

[9] H. Hindi and S. Boyd, "Analysis of linear systems with saturation using convex optimization," in Proceedings of the 37th IEEE Conference on Decision and Control (CDC '98), pp. 903-908, Tampa, Fla, USA, December 1998.

[10] W. P. Dayawansa, C. F. Martin, and S. Samelson, "Asymptotic stabilization of a generic class of three-dimensional homogeneous quadratic systems," Systems \& Control Letters, vol. 24, no. 2, pp. 115-123, 1995.

[11] N. Vulpe, "Characterization of the finite weak singularities of quadratic systems via invariant theory," Nonlinear Analysis. Theory, Methods \& Applications, vol. 74, no. 17, pp. 6553-6582, 2011.

[12] H. Gingold and D. Solomon, "On completeness of quadratic systems," Nonlinear Analysis. Theory, Methods \& Applications, vol. 74, no. 12, pp. 4234-4240, 2011.

[13] T. Hu and Z. Lin, Control Systems with Actuator Saturation: Analysis and Design, Birkhauser, Boston, Mass, USA, 2001.

[14] R. Genesio and A. Vicino, "Some results on the asymptotic stability of second-order nonlinear systems," IEEE Transactions on Automatic Control, vol. 29, no. 9, pp. 857-861, 1984.

[15] V. Kapila and K. Grigoriadis, Actuator Saturation Control, Marcel Dekker, New York, NY, USA, 2002.

[16] M. A. Krosnosel'skii and P. P. Zabreiko, Geometric Methods of Nonlinear Analysis, Springer, New York, NY, USA, 1984. 
[17] B. Zhou and G.-R. Duan, "A novel nested non-linear feedback law for global stabilisation of linear systems with bounded controls," International Journal of Control, vol. 81, no. 9, pp. 1352-1363, 2008.

[18] B. Zhou, W. X. Zheng, and G.-R. Duan, "An improved treatment of saturation nonlinearity with its application to control of systems subject to nested saturation," Automatica, vol. 47, no. 2, pp. 306-315, 2011.

[19] T. Alamo, A. Cepeda, and D. Limon, "Improved computation of ellipsoidal invariant sets for saturated control systems," in Proceedings of the 44th IEEE Conference on Decision and Control, and the European Control Conference (CDC-ECC '05), pp. 6216-6221, Seville, Spain, December 2005. 


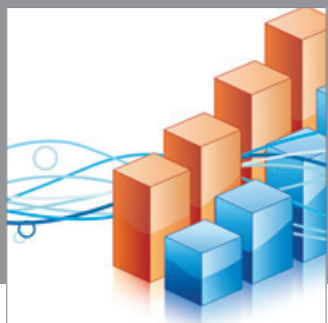

Advances in

Operations Research

mansans

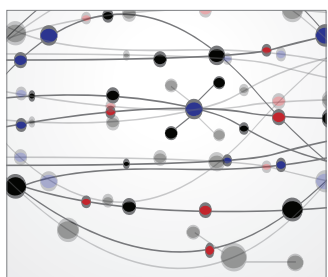

The Scientific World Journal
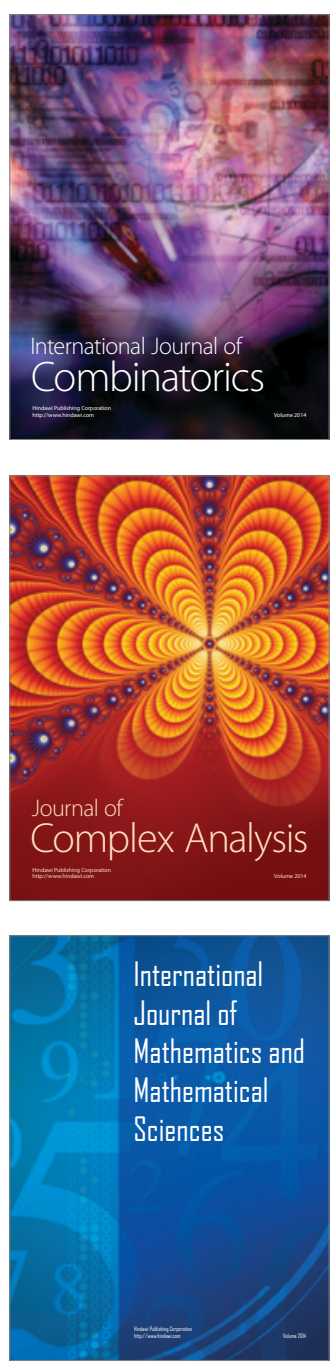
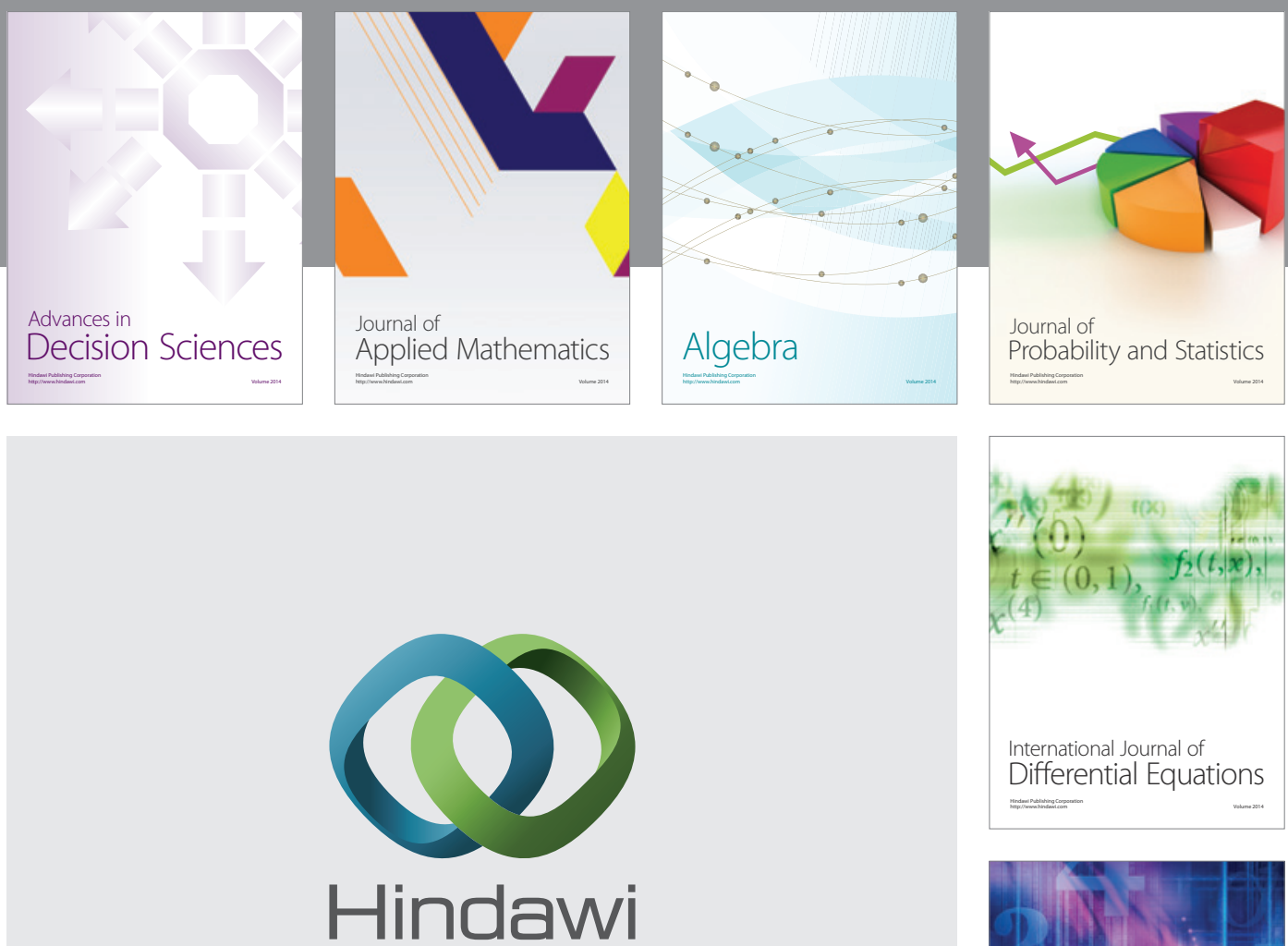

Submit your manuscripts at http://www.hindawi.com
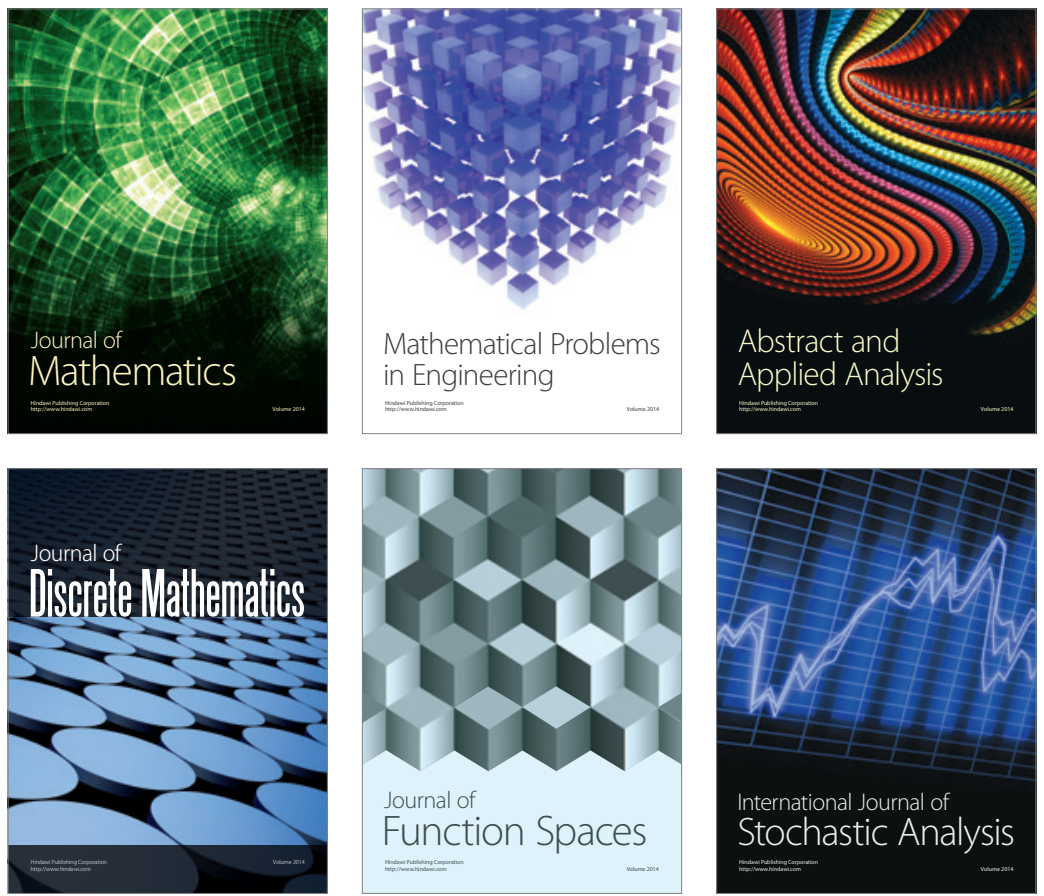

Journal of

Function Spaces

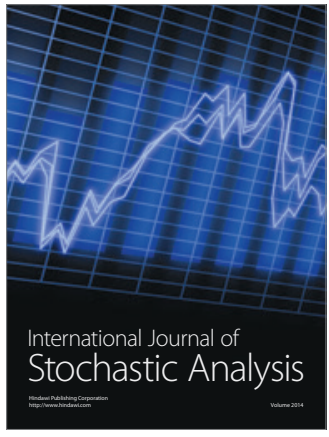

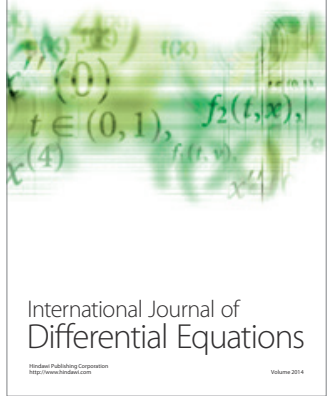
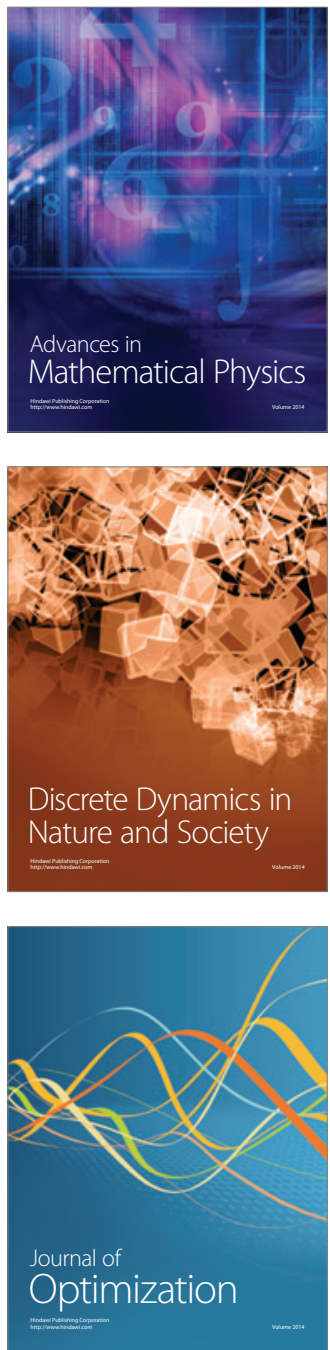